\title{
An IoMT system for health monitoring in athletes
}

\author{
Eduardo García Michel, Pedro C. Santana-Mancilla, Silvia B. Fajardo-Flores, \\ Laura S. Gaytan-Lugo, Víctor Hugo Pérez Andrade, Geraldyluz Amezcua \\ Cobián, Oscar Virgen Casillas, Sergio Alejandro Zaizar Fregoso
}

Published: 30 November 2020

\begin{abstract}
Continuous health monitoring in real-time has become essential to improve people's quality of life through medical prescription or personal control. Our goal is to develop a wearable IoMT device with real-time monitoring of heart rate and breathing patterns while an athlete performs physical exercise at high-intensity intervals. The wearable IoMT device incorporates vital signs sensors to record and display information in a mobile application, allowing users to track their health and receive an alert if the data exceeds normal parameters.
\end{abstract}

\section{Keywords:}

Athletes; Internet of Medical Things; Wearable Computing; High Intensity Interval Training.

\section{Introducción}

Llevar una vida sedentaria sin realizar actividad física puede ocasionar problemas de salud como diabetes mellitus, cáncer de colon, cáncer de mama y cáncer de pulmón [11].

La mejor forma de evitar una vida sedentaria es ejercitarse. En la actualidad, se ha popularizado un tipo de entrenamiento que se caracteriza por una etapa de ejercicio vigoroso con intervalos de descanso cortos; a esto se le conoce como Entrenamiento por Intervalos de Alta Intensidad (o por sus siglas en inglés HIIT). Además, los protocolos HIIT acortan el tiempo total dedicado a la actividad física y producen la misma carga de trabajo que una sesión regular de ejercitación [14,20].

El cuerpo humano presenta diferentes constantes fisiológicas, llamadas signos vitales, los cuales pueden ser monitoreados para seguimiento de la salud. La literatura médica considera como signos vitales esenciales al ritmo cardíaco, la presión arterial, el patrón respiratorio, la saturación de oxígeno y la temperatura, los cuales por [6] como:

García Michel, E., Santana-Mancilla, P., Fajardo-Flores, S., GaytanLugo, L., Pérez Andrade, V., Amezcua Cobián, G., Virgen Casillas, O., Zaizar Fregoso, S.

Universidad de Colima

egarcia24@ucol.mx,psantana@ucol.mx,medusa@ucol.mx, laura@ucol.mx,vperez6@ucol.mx, gamezcua1@ucol.mx, ovirgen0@ucol.mx, szaizar0@ucol.mx
- El ritmo cardíaco es la cantidad de latidos por minuto; este puede ser medido a través del pulso.

- La presión arterial se mide tradicionalmente a través de un esfigmomanómetro el cual convierte la presión ejercida sobre las paredes arteriales en milímetros de mercurio.

- El patrón respiratorio refleja la cantidad de inhalaciones y exhalaciones por minuto; y se refleja a través de cambios de volumen en el tórax de la persona.

- La saturación de oxígeno es un parámetro que estima la cantidad de oxígeno que es portado por los eritrocitos.

- La temperatura corporal refleja el balance entre la producción de calor por el cuerpo y sus mecanismos de pérdida del mismo.

Existen evidencias que sugieren que el esfuerzo físico puede ser medido basado en la frecuencia respiratoria, puesto que muestra una respuesta rápida al trabajo de recuperación durante el HIIT, mientras que la frecuencia cardiaca y la cantidad máxima de oxígeno muestran una respuesta retardada [15]. Esta respuesta rápida hace que la frecuencia respiratoria sea potencialmente adecuada para registrar el esfuerzo físico durante actividades intermitentes y la frecuencia cardiaca sea un buen complemento.

En este proyecto se busca dar seguimiento a la actividad física de una persona mientras realiza un entrenamiento HIIT por medio de un dispositivo vestible que incorpore sensores de frecuencia respiratoria y frecuencia cardiaca, el objetivo es que se trate de un dispositivo no invasivo, cómodo y fácil de usar para los atletas.

El monitoreo remoto de la salud se define como el cuidado de la preservación del estado de salud de un ser humano a través de la medición y la interpretación de señales biológicas, sin la interferencia con las actividades del mismo [8]. Los sensores actualmente comercializados para recabar las señales fisiológicas del cuerpo humano son costosos y su medición se ve altamente afectada por ruido, un tipo de interferencia dependiente de la forma en la cual se obtiene la medición deseada [4].

Los dispositivos de salud vestibles, son una tecnología emergente que permite el cuidado continuo ambulatorio de los signos vitales de un ser humano, dando soporte al diagnóstico médico. Para que un sensor clasifique como un dispositivo vestible debe ser de bajo consumo de energía, confiable, seguro, confortable y ergonómico [6].

Este concepto de dispositivos vestibles con sensores incorporados en una red de comunicación entra dentro del Internet de las Cosas (IoT). Cuando estos sistemas funcionan como tecnología de apoyo al cuidado de la salud, los involucrados en el proceso (médicos, cuidadores, entrenadores, etc.) pueden supervisar el estado de salud de forma remota en tiempo real, esto 
ha generado el concepto de Internet de las Cosas Médicas (IoMT por sus siglas en inglés, Internet of Medical Things), que es IoT adaptado al cuidado de la salud [18].

El monitoreo en tiempo real de los signos vitales será de gran utilidad ya que puede ayudar a optimizar el entrenamiento HIIT debido a que se estarán observando las constantes vitales del sujeto, por lo tanto, si su ritmo cardiaco o frecuencia respiratoria aumentan a niveles en donde pueda ser perjudicial para la salud, se le enviará una alerta con indicaciones para que realice una serie de pasos para estabilizarlos.

La parte de innovación de este proyecto consiste en que este monitoreo podrá ser capaz de encontrar patrones anormales de signos vitales, como probables causantes de eventos agudos al realizar la actividad física [7].

Este manuscrito es una versión extendida del artículo "Towards a Wearable Device for Monitoring Health of Athletes" publicado en la memoria de la IX Latin American Conference on Human Computer Interaction (CLIHC '19) [2].

\section{Método}

El presente trabajo se realizó usando el Diseño Centrado en el Usuario, con el objetivo de conocer, entender y asegurar que el producto final será de calidad y contará con la aceptación de los usuarios finales. Consiste en un proceso iterativo de cuatro etapas: especificar el contexto de uso, especificar requerimientos, producir soluciones de diseño y evaluación.

\subsection{Especificar el Contexto de Uso}

El contexto de nuestra aplicación se obtuvo en dos partes: revisión de literatura y entrevistas in situ en los gimnasios de la ciudad de Colima en México.

\subsubsection{Revisión de literatura}

Se realizó una exploración inicial de la literatura, reportada en [21], en dicha revisión se siguió la guía PRISMA con los siguientes pasos:

1. La definición de las palabras clave (se buscaron en idioma inglés) de búsqueda para información relacionada serían: intervalos de entrenamiento de alta intensidad (high intensity interval training, HIIT); respiraciones por minuto (respiration rate), dispositivo vestible (wearable device).

2. Investigación a través del motor búsqueda Google Académico. Se obtuvieron 5 mil 580 artículos, con los cuales se realizó una redefinición de los criterios de búsqueda.

3. Las primeras palabras clave parecieron se insuficientes, por lo que se redefinieron a las siguientes: entrenamiento (training); respiración por minuto (respiration rate); dispositivo vestible (wearable device); monitoreo respiratorio (breathing monitoring); sensor; cuidado de la salud (health care); intervalos de entrenamiento de alta intensidad (high intensity interval training, HIIT); no invasivos (non-invasive); sin patente (no patented).

4. Posteriormente, se establecieron parámetros de exclusión de artículos. Con esto se conformó la nueva base de datos de información, la cual fue utilizada para obtener el contexto del estado del arte.

Como conclusión de la revisión de literatura, se encontró que existe la tendencia hacia los dispositivos vestibles para monitorear los signos vitales de las personas. Además, se encontraron diversos escenarios que indican que el control de la respiración es bastante efectivo para lograr la recuperación de un cuerpo fatigado.

\subsubsection{Entrevistas}

Para lograr el entendimiento inicial de los usuarios, se entrevistó a ocho entrenadores y preparadores físicos del mismo número de gimnasios en la ciudad de Colima, donde los perfiles de los entrenadores eran Licenciados en Educación Física y de los cuales uno cuenta con una certificación para realizar entrenamiento en grupo. La entrevista se realizó con la finalidad de obtener información sobre si en los gimnasios se realizan mediciones de signos vitales en sus atletas, así como el uso que la dan a dicha información durante las sesiones de entrenamiento.

La primer pregunta indaga sobre el uso de entrenamiento tipo HIIT por los entrenadores. El 43\% de los entrevistados afirmaron que realizaban este tipo de entrenamiento (Figura 1).

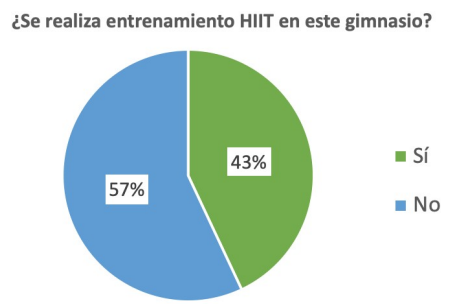

Figura 1. Porcentaje de entrenadores que realizan entrenamiento HIIT.

La manera en que los entrenadores lo hacen depende del gimnasio y la capacitación de cada uno de ellos. Por ejemplo, en uno de los gimnasios, el entrevistado afirmaba que realizaba el entrenamiento HIIT trabajando con el peso corporal de sus clientes mientras que en el resto de gimnasios trabajan con rutinas de levantamiento de pesas. En ambos tipos de entrenamientos la dinámica es similar y consiste en realizar aproximadamente un minuto de un entrenamiento exhaustivo seguido de 2 minutos entrenamiento ligero con la finalidad de no permitir que el cuerpo alcance un estado de descanso total. La importancia del descanso mientras haces el entrenamiento HIIT es para regular los niveles de glucosa permitiendo que el cuerpo recupere sus niveles de energía y con esto el usuario podrá tener energía para incrementar su intensidad en el entrenamiento [17].

Se preguntó a los entrenadores qué tipo de signos vitales de sus clientes obtienen y registran. Los principales datos que recolectan son: peso, edad y estatura, con la finalidad de dar un entrenamiento más personalizado y adecuado a sus cuerpos (Figura 2). Un bajo porcentaje de los entrevistados mide y recolecta datos de signos vitales durante los entrenamientos, mencionaron que miden la frecuencia cardíaca y los niveles de oxigeno en la sangre para evitar problemas respiratorios, mareos o debilidad. Los medios que usan para medir estos signos vitales son: pulsioxímetro, manual por medio de la carótida y dispositivo especializado. Ninguno de los entrevistados lleva el registro de los signos vitales, los usan para tener control durante el entrenamiento.

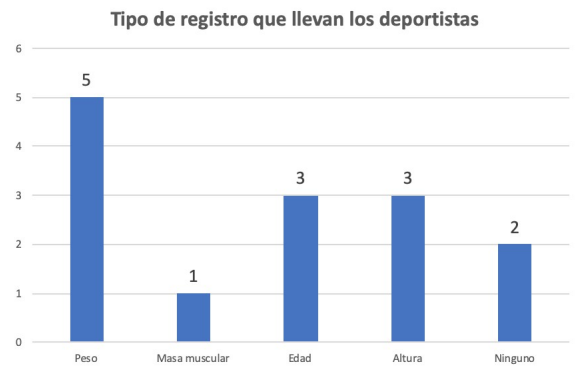

Figura 2. Datos físicos obtenidos por los entrenadores. 
También se indagó sobre el uso de dispositivos que monitorean signos vitales directamente por los clientes. Se encontró que una parte importante $(71 \%)$ de atletas monitorean por su cuenta sus signos vitales (Figura 3). El 57\% usan un smartwatch o una banda inteligente, el $14 \%$ mencionó que lo hacía sin mediación tecnológica, es decir contando el número de latidos por minuto a través de la carótida (Figura 3).

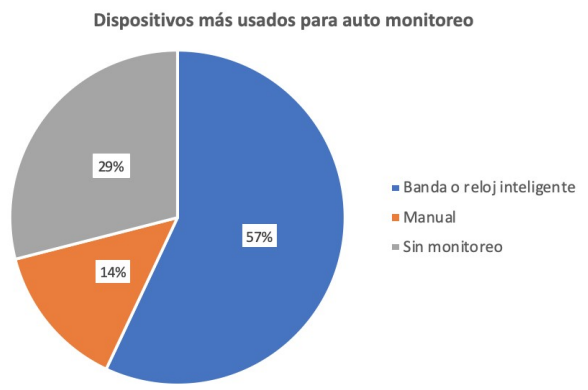

Figura 3. Medición de signos vitales por los atletas.

Los datos recolectados de las entrevistas nos permiten tener las siguientes conclusiones:

- El entrenamiento HIIT es usado en los gimnasios de la ciudad, pero no es el más común.

- Los que usan HIIT coinciden que se debe tener una rutina personalizada para cada atleta dependiendo de su edad, altura y peso.

- Durante el entrenamiento, el monitoreo de los signos vitales se realiza para verificar que tan efectivo es el entrenamiento en sus clientes y saber si el usuario resiste la intensidad de entrenamiento que le están aplicando o si es necesario reducir dicha intensidad.

- No todos los entrenadores se encargan de monitorear los signos vitales de sus clientes.

- La mayoría de los atletas monitorean sus propios signos vitales, utilizando sus propios dispositivos; estos clientes reportan a sus entrenadores sus signos vitales para obtener un entrenamiento personalizado.

- Se requiere apoyo de mediación tecnológica para automatizar el proceso de monitoreo y mantener un historial de los signos vitales de los atletas.

\subsection{Especificar Requerimientos}

Basado en el análisis de esta información, se obtuvieron las características que el sistema a desarrollar debe cumplir para dar apoyo a los deportistas que realizan entrenamiento HIIT.

- Debe ser un dispositivo no invasivo, el cual permita a los usuarios realizar sus entrenamientos sin interferir.

- Debe monitorear la frecuencia de respiración y el ritmo cardiaco.

- Debe ser de bajo consumo de energía y de bajo costo.

\subsubsection{Dispositivos vestibles}

Los datos obtenidos de las entrevistas nos muestran que los atletas utilizan dispositivos personales de monitoreo y en su mayoría usan de tipo pulsera. Es por eso, que se propone la creación de un brazalete para que no sea invasivo y sea consistente con lo que acostumbran a utilizar. La Figura 4 muestra el prototipo del brazalete [2].

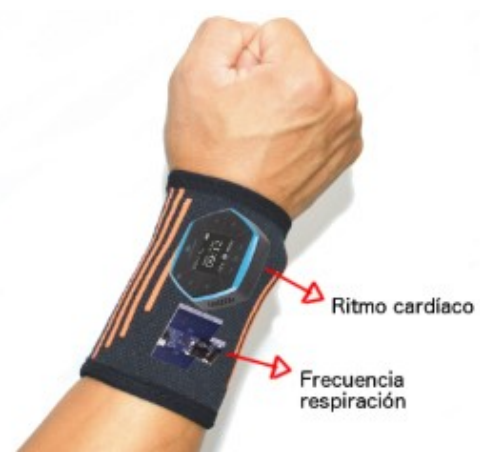

Figura 4. Prototipo IoMT para monitoreo de signos vitales.

\subsubsection{Medición del ritmo cardiaco}

Para realizar el monitoreo de la frecuencia cardíaca se ha seleccionado el dispositivo Hexiwear, el cual incluye sensores para medir variables biométricas tales como ritmo cardíaco $\mathrm{y}$ temperatura, además de contar con podómetro para el control de pasos, fue seleccionado porque tanto software como hardware son abiertos, además de contar con comunicación inalámbrica y ser eficiente energéticamente [9].

\subsubsection{Medición de la frecuencia de respiración}

Para el monitoreo de la frecuencia de respiración, se utilizará un sensor de bajo costo montado en chip llamado X4M200 (ver Figura 6). Dicho sensor contiene un algoritmo para la detección de la respiración de las personas por medio de radar [22]. Existe en la literatura evidencia suficiente que demuestra la fiabilidad de este sensor para medir la frecuencia respiratoria cercana a grado médico $[1,10,12,13]$

\subsubsection{Arquitectura del sistema}

La Figura 5 muestra la arquitectura del sistema.

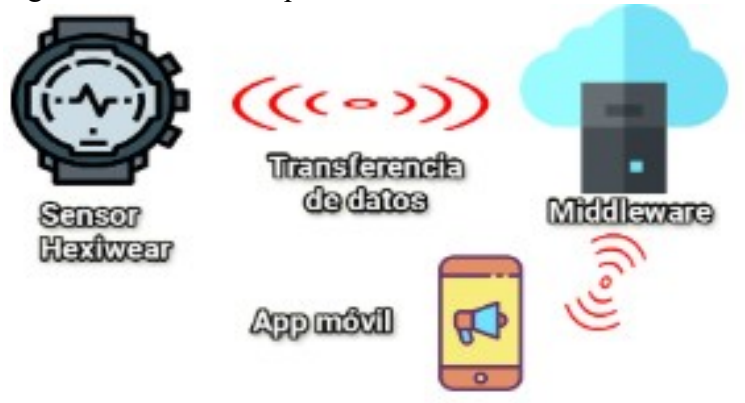

Figura 5. Arquitectura del sistema IoMT.

La arquitectura consiste en tres capas:

- Sensores

- Middleware

- Aplicación móvil

La primera capa consiste en el monitoreo de las constantes vitales por parte de los sensores en el brazalete; dichos datos se envían a la siguiente capa. La segunda capa es de software y se trata de un middleware que recolecta los datos de los sensores y obtiene el contexto para enviar la información y posibles alertas a la siguiente capa. La última capa consiste en una aplicación móvil, la cual permite visualizar la información del monitoreo de la salud en tiempo real, así como indicar las alertas que se generen durante el ejercicio. 
Dicha arquitectura permite el funcionamiento general del sistema IoMT propuesto, el cual se ilustra en la Figura 6 y se describe a continuación:

1. El usuario inicia su sesión de ejercicio.

2. Los sensores miden las constantes vitales de forma continua y lo envía al middleware.

3. Una vez que el middleware detecte una anomalía al analizar los datos, la clasificará.

4. Si la anomalía cae en una clasificación de riesgo, se envían alertas tanto al entrenador como a la aplicación móvil y al brazalete para instruir a la persona sobre qué debe de hacer para normalizar sus signos vitales.

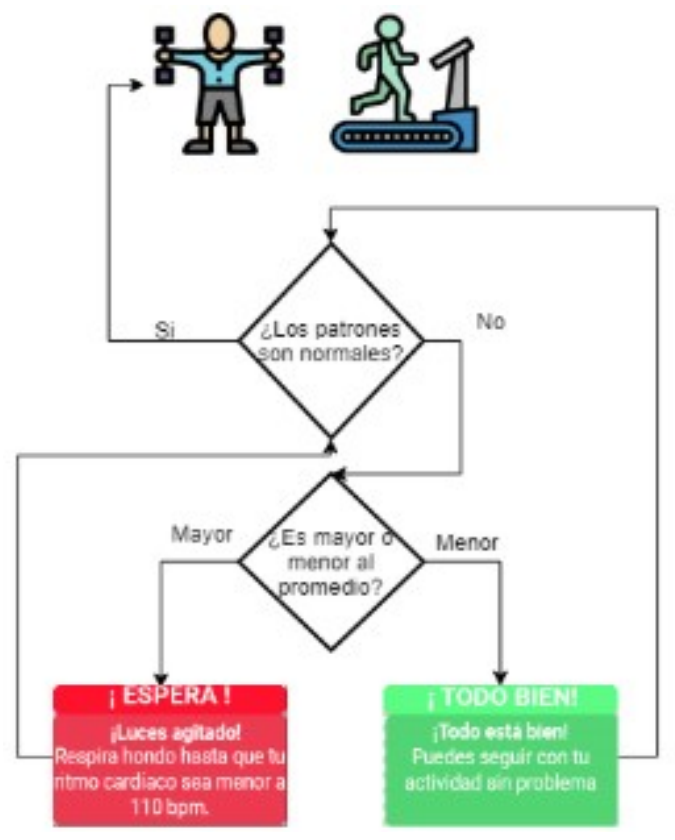

Figura 6. Funcionamiento general del sistema IoMT.

\subsection{Producir Soluciones de Diseño}

Con los requerimientos completos y la arquitectura propuesta diseñada, se crearon dos prototipos de mediana fidelidad, uno del brazalete (ver Figura 4), que en esta primera iteración incluye solamente el sensor de ritmo cardíaco, y otro de la aplicación móvil.

La Figura 7 muestra tres de las principales pantallas de la aplicación. Cuando el middleware detecta que los datos de los sensores se encuentran fuera del rango normal lanza una alerta (ver Figura 8) para que el usuario tome un descanso de recuperación. Durante el tiempo de reposo el monitoreo sigue en tiempo real desde el dispositivo, cuando se detecta que los valores regresan a la normalidad se le notifica que puede continuar (ver Figura 9).

\subsection{Evaluación}

En esta etapa del proyecto, se realizó una evaluación de la experiencia de usuario y aceptación de uso, para asegurar que los requerimientos identificados fueron incluidos de manera que sean fáciles de usar y útiles para los atletas.

Se aplicaron dos instrumentos de evaluación, la Escala de Usabilidad de Sistemas (SUS por sus siglas en inglés) y el Modelo de Aceptación de la Tecnología (TAM por sus siglas en inglés). El SUS es un instrumento muy útil para evaluar la percepción de usabilidad de los usuarios de una forma sencilla y rápida [3]. El TAM, consiste en un modelo que ha sido probado y validado ampliamente y se utiliza para predecir la aceptación de una tecnología [5].

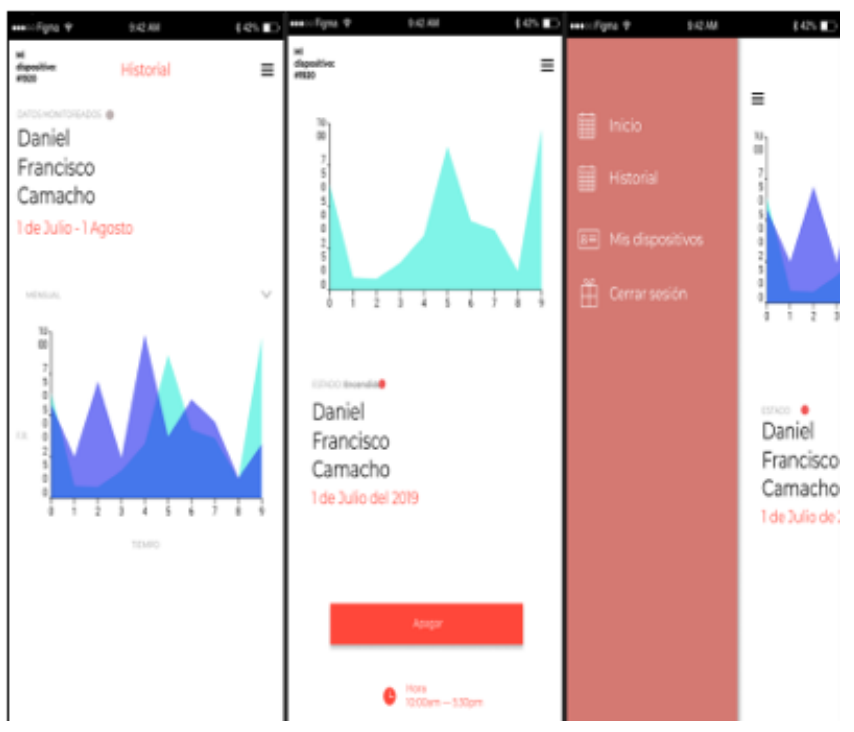

Figura 7. (a) Pantalla de monitoreo activo; (b) Opciones de configuración; y (c) Pantalla del historial

¡Nos estamos cansando!

Toma un descanso de 1 minuto y continúa con tu trabajo

Figura 8. Alerta para tomar un descanso del entrenamiento.

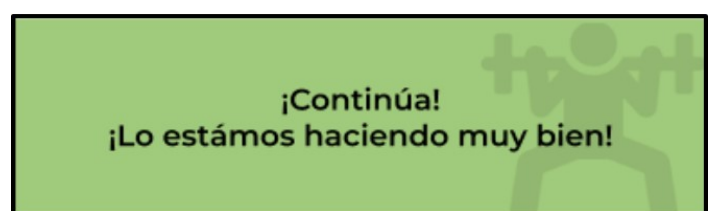

Figura 9. Notificación de que se puede continuar con el ejercicio.

\subsubsection{Participantes}

Jakob Nielsen propone [16] evaluar en varias etapas del proyecto (por ejemplo las iteraciones en el Diseño Centrado en el Usuario) con cinco usuarios, y que esto puede detectar la mayoría de los problemas de usabilidad. Siguiendo dicha recomendación, para esta etapa del proyecto se seleccionaron dos grupos de cinco usuarios de tipo deportistas, todos son clientes de gimnasios. Las muestras se seleccionaron por muestreo por conveniencia, por la disponibilidad de los participantes. La única condición para participar es que el entrenamiento fuera de tipo HIIT, la evaluación se realizó directamente en las instalaciones de los gimnasios con las rutinas de ejercicio que ya tenían programadas para ese día de entrenamiento.

\subsubsection{Proceso}

Primero, se explicó el prototipo a los usuarios. Después, se les asignó una lista de actividades que debían realizar con el sistema 
móvil usando el dispositivo vestible IoT. Finalmente, se pidió a los participantes que llenaran los instrumentos SUS y TAM, para medir su percepción sobre la usabilidad y la aceptación hacia el sistema IoT de monitoreo a la salud mientras practican entrenamiento HIIT.

\section{Resultados}

\subsection{Escala de Usabilidad de Sistemas}

La evaluación SUS dio una calificación de 77.5, que de acuerdo con la teoría [19], puede ser considerado como muy usable. La Figura 12 muestra la distribución de las frecuencias de cada participante en la evaluación. En ella se puede observar que, de forma individual, los usuarios evaluaron al sistema con una percepción positiva.

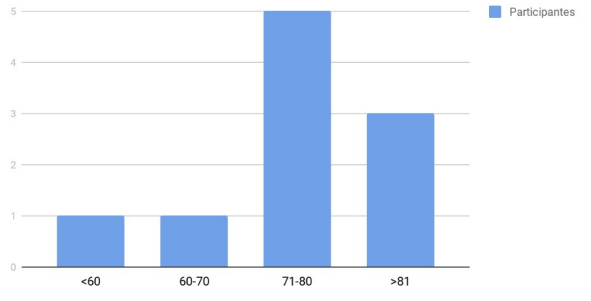

Figura 12. SUS distribuciones de frecuencia.

\subsection{Modelo de Aceptación de la Tecnología}

Tomando en cuenta solamente las respuestas "Muy de acuerdo" y "Totalmente de acuerdo", el instrumento TAM generó los resultados siguientes.

En la dimensión referente a la "Percepción de facilidad de uso" (ver Figura 13), los ítems "Fácil de usar" y "Fácil de entender" tuvieron el $100 \%$ de respuestas positivas por parte de los usuarios, mientras que el $90 \%$ dijo que era claro y entendible y se encontraba la información fácilmente.

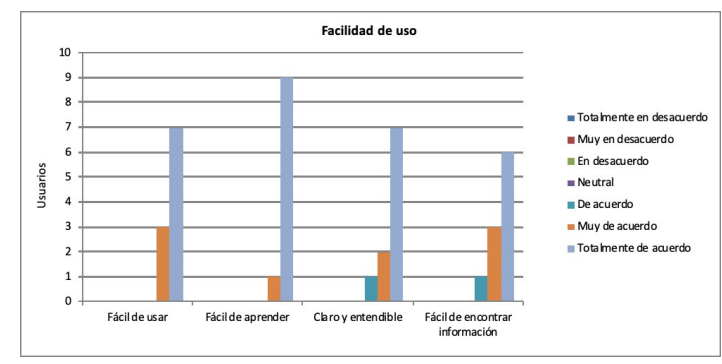

Figura 13. TAM facilidad de uso.

En el área de la percepción de utilidad, los resultados obtenidos fueron, el $70 \%$ cree que es muy eficiente y que mejoraría su desempeño, el $80 \%$ piensa que mejoraría su productividad y finalmente el $90 \%$ creen que les sería útil este sistema (ver Figura 14).

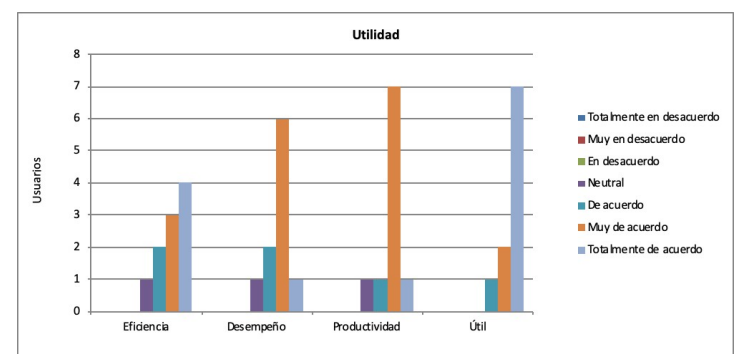

Figura 14. TAM utilidad.
La dimensión relacionada a la actitud de los atletas respecto al uso del software fue la mejor evaluada (ver Figura 15), ya que se obtuvieron porcentajes altos de respuestas positivas, el $90 \%$ se sienten seguros de usarlo, mientras que el 100\% mantienen una actitud favorable sobre usarlo, creen que es una buena idea y que era beneficioso usarla.

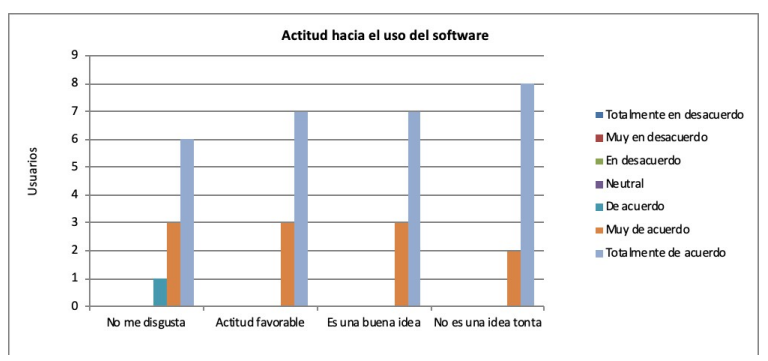

Figura 15. TAM actitud hacia el uso del software.

En la última dimensión del TAM "Intención de uso", el 70\% tienen la intención de utilizar el dispositivo IoT y la aplicación, el $80 \%$ de lo volverían a utilizar, mientras que el $60 \%$ tienen la intención de utilizarlo en sus entrenamientos (ver Figura 16).

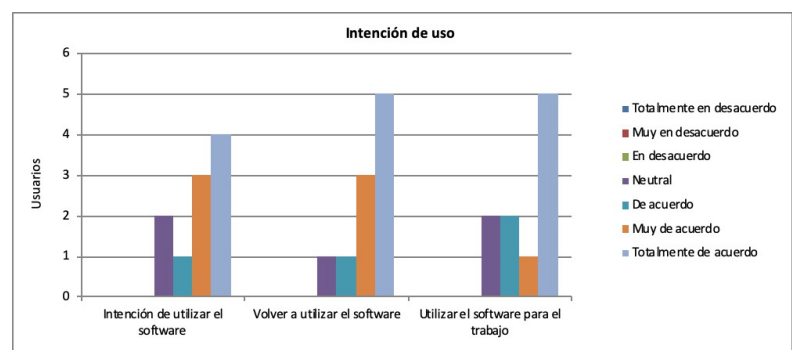

Figura 16. TAM intensión de uso.

\section{Conclusiones}

Se presenta la propuesta de diseño de un sistema IoT para monitoreo de signos vitales en deportistas mientras realizan entrenamiento HIIT, el sistema consiste en un dispositivo vestible con sensores de variables fisiológicas y una aplicación móvil para visualización y alertas.

El diseño fue participativo con entrenadores de gimnasios a los que se les aplicó una entrevista, y se complementó con una revisión de literatura. La implementación demostró que es factible realizarlo y se alinea al paradigma IoT.

Los resultados de la evaluación de experiencia de usuario y aceptación de uso fueron positivos. Lo anterior parece indicar que el sistema propuesto puede ayudar a disminuir los riesgos de salud al realizar entrenamiento HIIT.

Finalmente, se propone crear una nueva iteración del proceso. Dicha nueva iteración incluiría la utilización del sensor de frecuencia respiratoria basada en radar. Una vez completado dicho prototipo se planea realizar una evaluación de larga duración en gimnasios para validar la propuesta de apoyo a la salud de los deportistas.

\section{Agradecimientos}

Los autores agradecen a los gimnasios por la autorización para realizar la evaluación en sus instalaciones, y a todas las personas que participaron como sujetos durante el estudio. Agradecemos los comentarios y retroalimentación recibidos por cuatro revisores anónimos que nos ayudaron a mejorar el documento. 


\section{Referencias}

[1] Ali, K., Alloulah, M., Kawsar, F., and Liu, A. X. On Goodness of WiFi based Monitoring of Vital Signs in the Wild. (Mar. 2020). arXiv: 2003.09386.

[2] Andrade, V. H. P., Santana-Mancilla, P. C., Fajardo-Flores, S. B., Michel, E. G., Gaytan-Lugo,' L. S., and RodríguezOrtiz, M. A. Towards a wearable device for monitoring health of athletes. In Proceedings of the IX Latin American Conference on Human Computer Interaction (New York, NY, USA, 2019), CLIHC '19, Association for Computing Machinery.

[3] Brooke, J. Sus: a quick and dirty usability. Usability evaluation in industry (1996), 189.

[4] Dasgupta, A., Chakraborty, S., Sengupta, A., and Routray, A. Active Sensors for the Acquisition of Physiological Signals. IEEE, pp. 1-3.

[5] Davis, F. D., Bagozzi, R. P., and Warshaw, P. R. User acceptance of computer technology: a comparison of two theoretical models. Management science 35, 8 (1989), 9821003.

[6] Dias, D., and Paulo Silva Cunha, J. Wearable Health Devices-Vital Sign Monitoring, Systems and Technologies. Sensors 18, 8 (July 2018), 2414.

[7] Gonzalez, M., García, M., Exposito, I., and Garrido, R. Patrones de desaturación ergoespirométricos en función de la edad. Revista Internacional de Medicina y Ciencias de la Actividad Física y del Deporte 5, 18 (2005), 100-117.

[8] Hermens, H. J., and Vollenbroek-Hutten, M. M. Towards remote monitoring and remotely supervised training. Journal of Electromyography and Kinesiology 18, 6 (Dec. 2008), 908919.

[9] Hexiwear. IoT and wearables development platform, 2018.

[10] Ivanovs, A., Nikitenko, A., Di Castro, M., Torims, T., Masi, A., and Ferre, M. Multisensor Low-Cost System for Real Time Human Detection and Remote Respiration Monitoring. IEEE, pp. 254-257.

[11] Javier Varo Cenarruzabeitia, J., Martínez Hernandez, J. A., and Martínez Gonzalez, M. A. Beneficios de la actividad física y riesgos del sedentarismo. Medicina Clínica 121, 17 (Jan. 2003), 665-672.
[12] Kumar, D., Sarkar, A., Kerketta, S. R., and Ghosh, D. Human Activity Classification Based On Breathing Patterns Using IRUWB Radar. IEEE, pp. 1-4.

[13] Ma, Y., Liang, F., Wang, P., Yin, Y., Zhang, Y., and Wang, J. Research on Identifying Different Life States Based on the Changes of Vital Signs of Rabbit under Water and Food Deprivation by UWB Radar Measurement. IEEE, pp. 397403.

[14] Medbo, J. I., and Tabata, I. Relative importance of aerobic and anaerobic energy release during short-lasting exhausting bicycle exercise. Journal of Applied Physiology 67, 5 (Nov. 1989), 1881-1886

[15] Nicolo, A., Montini, M., Girardi, M., Felici, F., Bazzucchi, I., and Sacchetti, M. Respiratory Frequency as a Marker of Physical Effort During High-Intensity Interval Training in Soccer Players. International Journal of Sports Physiology and Performance 15, 1 (Jan. 2020), 73-80.

[16] Nielsen, J. Why you only need to test with 5 users, 2000.

[17] Peinado, A. B., Rojo-Tirado, M. A., and Benito, P. J. El azúcar y el ejercicio físico: su importancia en los deportistas. Nutrición hospitalaria 28, 4 (2013), 48- 56.

[18] Santana-Mancilla, P. C., Anido-Rifon, L. E., ContrerasCastillo, J., and Buenrostro-Mariscal, R. Heuristic evaluation of an IoMT system for remote health monitoring in senior care. International Journal of Environmental Research and Public Health 17, 5 (2020).

[19] Sauro, J. Measuring usability with the system usability scale (sus), 2011.

[20] Terada, S., Yokozeki, T., Kawanaka, K., Ogawa, K., Higuchi, M., Ezaki, O., and Tabata, I. Effects of high-intensity swimming training on GLUT-4 and glucose transport activity in rat skeletal muscle. Journal of Applied Physiology 90, 6 (June 2001), 2019-2024.

[21] Virgen, O., Fregoso, S. A. Z., Fajardo-Flores, S. B., Cobian, G. A., Santana-Mancilla, P. C., and Gaytan-Lugo, L. S. Health monitoring with wearable devices when performing physical exercise: An initial exploration of the state of the art. In Proceedings of the IX Latin American Conference on Human Computer Interaction (New York, NY, USA, 2019), CLIHC '19, Association for Computing Machinery.

[22] XeThru. X4M200 respiration sensor, 2018.

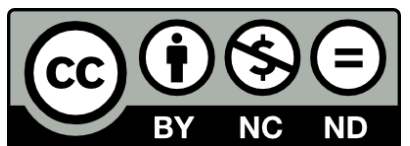

(c) 2020 by the authors. This work is licensed under the Creative Commons AttributionNonCommercial-NoDerivatives 4.0 International License. To view a copy of this license, visit http://creativecommons.org/licenses/by-nc-nd/4.0/ or send a letter to Creative Commons, PO Box 1866, Mountain View, CA 94042, USA. 